\author{
Military Technical College \\ Kobry El-Kobbah, \\ Cairo, Egypt
}

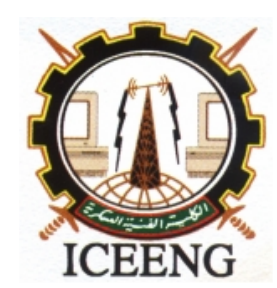

\title{
Adaptive GPS Tracking Loop Bandwidth Tuning Using Fuzzy Control and Inertial Aiding for Missile Applications
}

\begin{abstract}
By
Ahmed M. Kamel *

Abstract:

Autonomous Navigation Systems (ANS) used in ballistic or cruise missiles are mostly dependent on very high grade Inertial Navigation System (INS). INS is a dead reckoning system and its navigation solution always drifts with time from the correct one. Using aiding from other navigation sensors such as Global Positioning System (GPS) can help getting much better navigation solution and can allow dependence on lower grade INS. Data fusion from both INS and GPS can be done through an Extended Kalman Filter (EKF) either by fusing navigation solution from both sensors in a loosely coupled (LC) technique, or by using Doppler measurements aiding to aid INS or both INS and GPS as in tightly coupled (TC) or tightly coupled with aiding (TCA) technique which is sometimes called Ultra-Tight (UT) integration. GPS usage has limitations in terms of missile high dynamics and signal interference. The GPS receiver requirements to avoid these problems are conflicting. The Phase Lock Loops (PLLs), used to track GPS signals, are required to have a bandwidth as narrow as possible to reduce the impact of signal interference. On the contrary, the loop bandwidth has to be as wide as possible to accommodate high signal dynamics. To solve this contradiction, a new adaptive GPS tracking loop bandwidth tuning algorithm is designed based on integrated INS/GPS through an EKF linked to a Fuzzy Logic Controller (FLC). This controller senses the vehicle dynamics and the jamming signal conditions and decides the bandwidth value according to those inputs. The Adaptive Fuzzy Ultra Tight (AFUT) GPS/INS integration architecture has been developed and assessed by simulation using SPIRENT GSS7700 GPS hardware simulator showing that it provides a significant anti-jam and dynamics margins over an INS/GPS with fixed tracking bandwidths in addition to the ability of using lower grade and cheaper inertial sensors.
\end{abstract}

Keywords:

GPS, INS, Interference, High dynamics, Fuzzy, Kalman Filter

* Position, Location and Navigation (PLAN) Group

Department of Geomatics Engineering

Schulich School of Engineering

University of Calgary, Alberta, Canada 


\section{Introduction:}

Any Inertial Measuring Unit (IMU) composes of number of angular rate sensors (Gyroscopes) and acceleration sensors (Accelerometers). When an IMU is supplied by a navigation processor it is called then an Inertial Navigation System (INS). INS usage for autonomous navigation purposes has been widely used since decades. New technologies made it suitable to replace the huge size of old mechanical sensors with very small Micro Electro Mechanical Sensors (MEMS). More information about inertial sensors evolution can be found in [1]. Most of the errors accompanied with inertial measurements such as bias, drift, and scaling factor can be well defined, characterized, and nearly eliminated through the navigation processing.

Errors such as thermal noise and clock drift are hardly reduced and can cause a big deviation from correct navigation solution in a very small period of time. Inertial measurements from Gyros and accelerometers are used through a series of integrations, coordinate transformations, and compensations to drive a navigation solution that can be well understood by a navigator on earth and is defined by easting and northing coordinate frames. This process which is known in literature as "The mechanization equations" can be illustrated in blocks as shown in Figure (1) [2]. By setting the initial position; IMU measurements can be translated into sequence of positions and velocities estimated upon their previously determined values and that is the definition of a dead reckoning system.

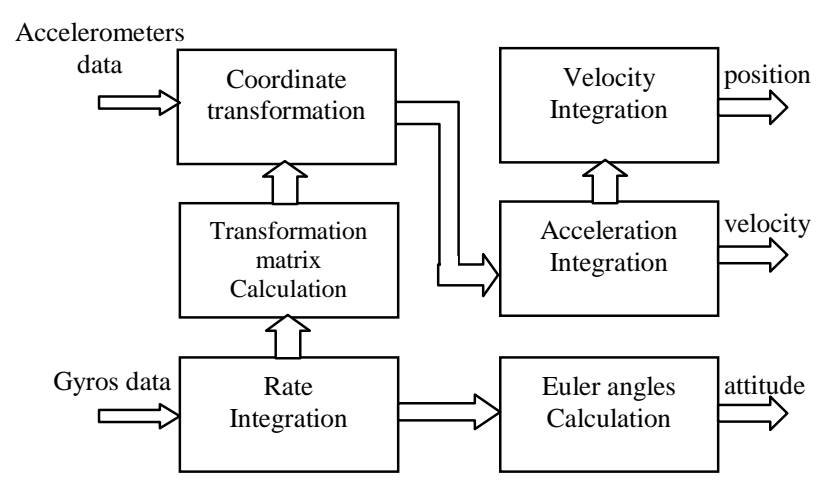

Figure (1): Mechanization blocks

As a contrary to INS, GPS is a single point positioning system that will not lead to a deviating navigation solution and can achieve accurate positioning within few meters or centimeters from true position [3]. Dependence on standalone GPS especially for 
military applications is not preferable due its slow data rate comparing to INS and its vulnerability against Radio Frequency (RF) interference. Data fusion for GPS and INS measurements can lead to a much better navigation solution evolved by avoiding disadvantages of either system.

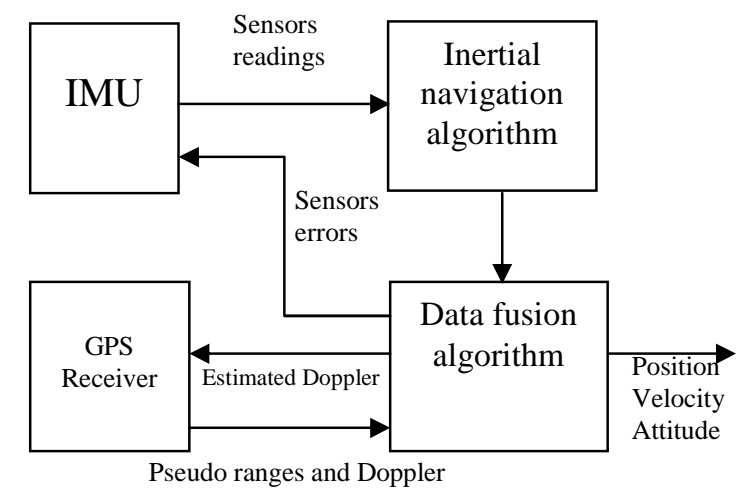

Figure (2): GPS/INS ultra tight integration

Several integration techniques have been studied well in the literature [4]. The technique adopted in this work is the tightly coupled with aiding (TCA) or it sometimes called Ultra Tight (UT) integration [5], which uses Doppler measurements to update Doppler, calculated within the EKF sequence. A general block diagram that describes UT integration is shown in Figure (2).

Although that using GPS tracking Doppler measurements for aiding and integration with INS is much better than using GPS calculated position or velocity (as in loosely coupled systems) due to correlated errors in the latter, it is very hard to fuse GPS and INS Doppler due to related noise effects.

Doppler frequency estimation of the carrier signal using INS can be expressed simply as the velocity of the receiver relative to the satellite, projected onto the line-of-sight (LOS) vector. This relationship is expressed in equation (1):

$f_{\text {Doppler }}=\frac{1}{\lambda}\left(\bar{v}_{r x}-\bar{v}_{s a t}\right) \cdot \bar{u}_{\text {sat }}$

where

$\bar{u}_{\text {sat }}=$ Unit LOS vector from receiver to satellite

$\bar{v}_{\text {sat }}=$ Velocity of satellite

$\bar{v}_{r x}=$ Velocity of receiver antenna

$\lambda \quad=$ Wavelength of carrier at L1 frequency 
The components of equation (1) can be computed in any Earth fixed reference frame, such as Earth-Centered-Earth-Fixed (ECEF) or local East-North-Up (ENU). The transformation matrix between these two reference frames is easily computed as a function of latitude and longitude. The computation of the ECEF satellite velocity from ephemeris data is a well-known procedure, described in [6].

INS calculated signal Doppler quality is dependent on how accurate INS velocity is calculated which in turn depends on INS grade, and its errors can grow up dramatically and unbounded.

GPS estimated signal Doppler quality is dependent on noise embedded in the signal. Narrow bandwidth Phase Lock Loop (PLL), which is the main signal tracking loop in the tracking system, is then preferred to be chosen to decrease the noise and RF interference effect on the GPS signal. Narrow bandwidth will decrease the ability to track signal dynamics which can be at high level in the case of missiles in concern. A detailed discussion on GPS tracking loop design and requirements is in next section.

\section{$\underline{\text { 2.GPS Tracking Loop Design and Requirements }}$}

GPS L1 signals are constructed of three components: carrier, spreading code and navigation data. To successfully demodulate the navigation data from the received signal, an exact carrier wave replica has to be generated and, to this end, Phase and Frequency Lock Loops (PLL and FLL) are generally used. Figure (3) shows the basic block diagram of a standard PLL (Farrell 2008). The two first multiplications are required to wipe off the input signal carrier and Pseudo-Random Noise (PRN) code. A local replica of the PRN code is provided by the Delay Lock Loop (DLL) and is used to remove the PRN sequence from the incoming signal. The loop discriminator is used to estimate the phase error between local and incoming carrier. The discriminator output, which represents the phase error, is then filtered and used to tune the Numerically Controlled Oscillator (NCO), which adjusts the frequency of the local carrier wave. In this way, the local carrier wave tends to be a precise replica of the input signal carrier.

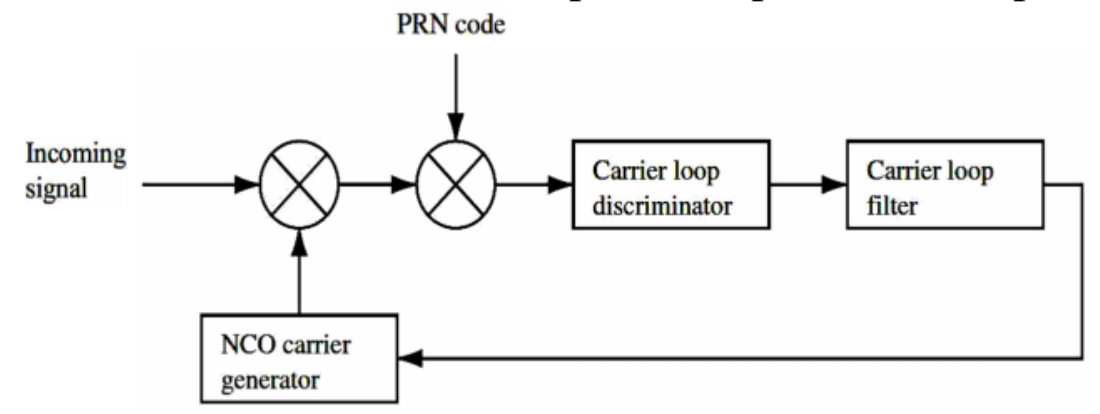

Figure (3): Basic PLL block diagram 
The design of PLLs has proven to be a challenging task, particularly if the receiver is affected by high dynamics or the input signal is very noisy. It is therefore desirable to provide robust algorithms for the PLL design.

FLLs are more resilient against signal dynamics and produce accurate velocity measurements. PLLs however also provide signal phase information; leading to a simplified data demodulation process as compared to FLLs. PLLs filter order and bandwidth are the two major parameters that affect the receiver capability to work under higher dynamics. The objective of the loop filter is to reduce noise in order to produce estimate of the original signal at its output. The loop filter order and bandwidth also determine the loop filter's response to signal dynamics. Table (1) summarizes these filter characteristics and provides the relations between loop order, bandwidth, and expected steady state error [3].

The loop filter natural radian frequency $\omega_{\text {op }}$ is computed from the loop filter bandwidth $B_{n p}$ value which is selected by the designer, and $R$ is the line of site range to the satellite. For high dynamic scenarios a $3^{\text {rd }}$ order PLL is usually used as it is only sensitive to acceleration jerks. Higher order PLLs can produce system instability and greater noise level. The loop filter of a typical $3^{\text {rd }}$ order PLL is shown in Figure (34) [3], where T is the update period of the loop.

Table (1): Loop Filter Characteristics

\begin{tabular}{|l|l|c|l|}
\hline $\begin{array}{l}\text { Loop } \\
\text { order }\end{array}$ & Bandwidth (Hz) & $\begin{array}{l}\text { Dynamic stress steady } \\
\text { state error }\end{array}$ & Sensitivity \\
\hline $1^{\text {st }}$ & $B_{n p}=0.25 \omega_{o p}$ & $\frac{d R / d t}{\omega_{o p}}$ & Velocity stress \\
\hline $2^{\text {nd }}$ & $B_{n p}=0.53 \omega_{o p}$ & $\frac{d R^{2} / d t^{2}}{\omega_{o p}^{2}}$ & Acceleration stress \\
\hline $3^{\text {rd }}$ & $B_{n p}=0.7845 \omega_{o p}$ & $\frac{d R^{3} / d t^{3}}{\omega_{o p}^{3}}$ & Jerk stress \\
\hline
\end{tabular}

All the gains shown in Figure (4) are design parameters and function of loop bandwidths $\mathrm{B}_{\mathrm{np}}$ as reported in Table (2). 
Table (2): $3^{\text {rd }}$ Order PLL loop filter gains

\begin{tabular}{|c|l|l|}
\hline$\omega_{o p}$ & $a_{3} \omega_{o p}^{2}$ & $b_{3} \omega_{o p}$ \\
\hline$\frac{B_{n p}}{10.1845}$ & $1.1 \omega_{o p}^{2}$ & $2.4 \omega_{o p}$ \\
\hline
\end{tabular}

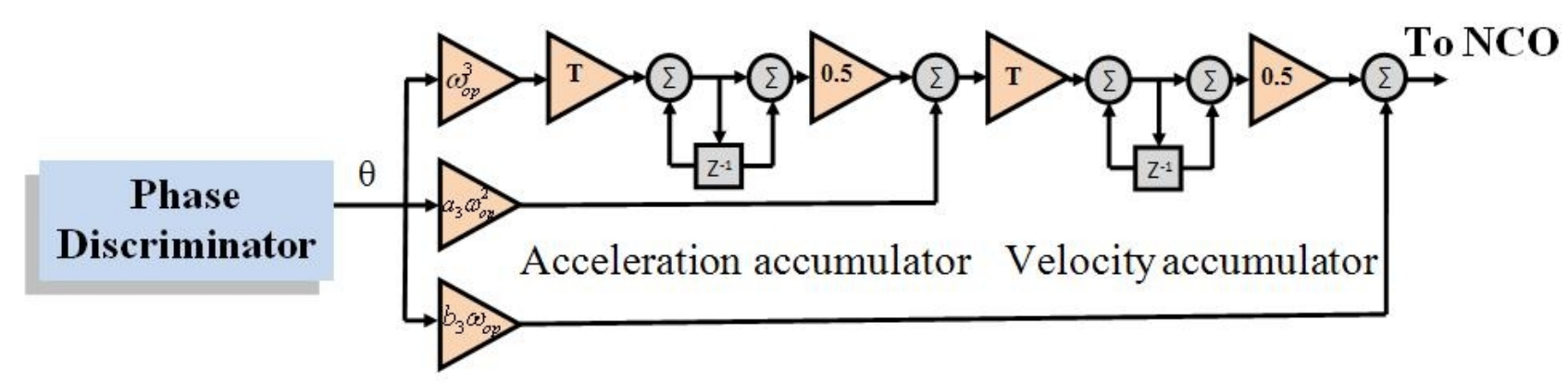

Figure (4): $3^{\text {rd }}$ order PLL loop filter

GPS measurement errors and tracking thresholds are closely related because the receiver loses lock when the measurement errors exceed a certain boundary [3]. Due to the non linearity of PLLs, only simulations of the GPS receiver under combined dynamic and signal-to-noise (SNR) conditions will determine the true tracking performance. However, rules of thumb can be used based on equations that predict the measurement errors of the tracking loops. The dominant sources of phase error in a GPS receiver PLL are phase jitter and dynamic stress error. The 3- sigma values of this PLL error can be computed as shown in Equation (2).

$3 \sigma_{P L L}=3 \sigma_{j}+\theta_{e} \leq 45(\mathrm{deg})$

Where

$\sigma_{j}=1$-segma phase jitter from all sources except dynamic stress error

$\theta_{e}=$ dynamic stress error in the PLL tracking loop. 


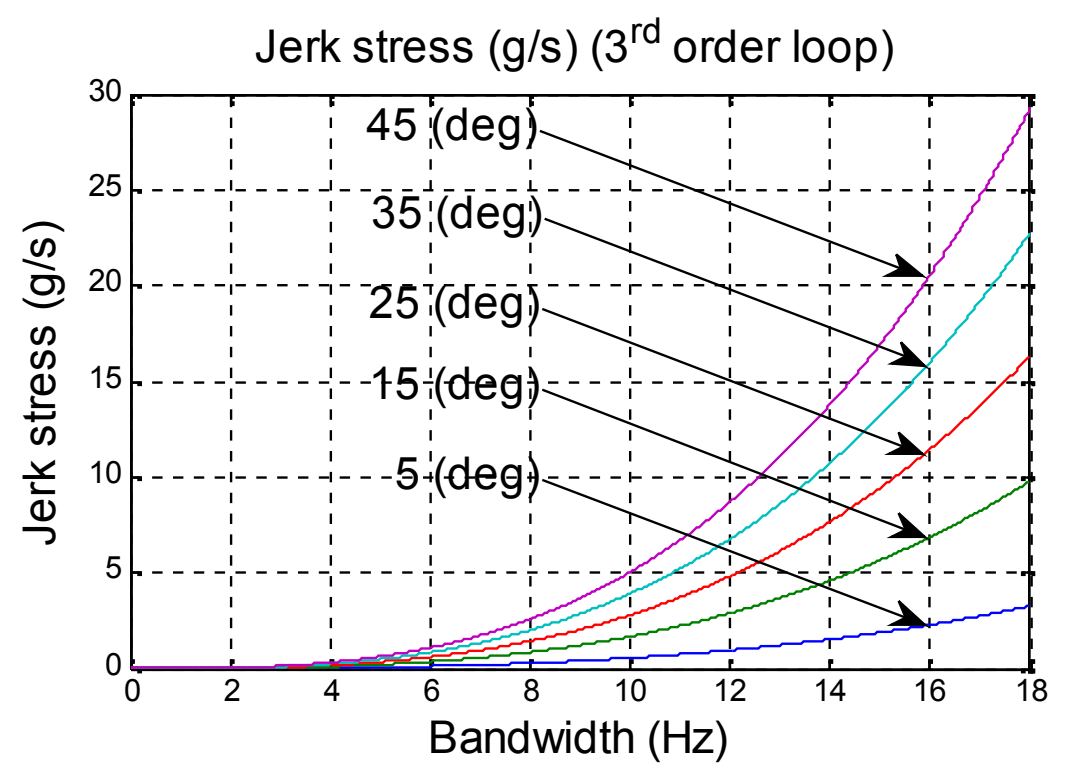

Figure (5): Relation between bandwidth and maximum allowable jerk stress at different phase tracking errors

Using natural frequency and bandwidth values for $3^{\text {rd }}$ order loop stated in Table (1) and assuming PLL dynamic stress error to take several values $\{5,15,25,35,45$ (deg) $\}$ (neglecting phase jitter), the relation between the maximum allowed jerk stress and bandwidth is calculated and illustrated in Figure (5). It can be seen that as long as the bandwidth increases, the capability of accepting higher dynamics is increasing which is one of the main points that is taken into consideration when designing GPS receiver for high dynamics applications.

Phase jitter $\left(\sigma_{j}\right)$ is mainly affected by thermal noise. The one sigma thermal noise jitter of this signal, when using a standard tracking loop, can be calculated as follows [7]:

$$
\sigma_{R}=\frac{360}{2 \pi} \sqrt{\frac{B_{n p}}{C / N o}\left(1+\frac{1}{2 T C / N o}\right)}(\mathrm{deg})
$$

where $\sigma_{\mathrm{R}}(\mathrm{deg})$ is the thermal noise standard deviation, $\mathrm{B}_{\mathrm{np}}(\mathrm{Hz})$ is the carrier loop noise bandwidth, $\mathrm{T}(\mathrm{s})$ is the predetection integration time and $\mathrm{C} / \mathrm{No}(\mathrm{dB}-\mathrm{Hz})$.

The response of a GPS receiver to different signal-to-noise levels depends on the code and carrier (phase/ frequency) tracking loop bandwidths. However there is a trade-off between noise resistance and response to dynamics. Narrow bandwidth tracking loops are more resistant to noise, that makes them suitable for moderate jamming environments. Wide bandwidth tracking loops are more responsive to dynamics. Thus, tracking loop bandwidth requirements for GPS receivers are conflicting. One possible 
solution is to adapt the tracking loop bandwidth to the receiver measured carrier-powerto-noise density ratio $(\mathrm{C} / \mathrm{No})$ and receiver dynamics. In the case of low jamming, a wide bandwidth can be used to cope with high dynamics. When the jamming is increased to near the loop threshold, i.e. the minimum equivalent $\mathrm{C} / \mathrm{No}$ that the loop can sustain without losing lock, the bandwidths of both code and carrier tracking loops are gradually reduced to enable higher jamming mitigation efficiency. But this adaptation to jamming reduces the tolerance to dynamics and a trade-off between dynamics and jamming rejection has to be found. More details about adaptation system design and methodology will be discussed in the next section.

\section{$\underline{\text { 3. Design of Adaptive Fuzzy Ultra Tight (AFUT) GPS/INS Integration Architecture }}$}

To tune the PLL bandwidth according to jamming signal and missile dynamics, a special purpose fuzzy processor is designed. More details on fuzzy control theory can be found in [8]. The fuzzy processor accepts two inputs which are missile acceleration in G's coming from the INS navigation processor and jamming signal level $J_{S}$ calculated in the PLL tracking loop.

The output of the fuzzy processor is the required PLL filter bandwidth which in turns changes the filter gain values reported in Table (1).

The architecture of the Adaptive Fuzzy Ultra Tight Coupled (AFUT) GPS/INS integration algorithm is shown in Figure (6). Bandwidth tuning fuzzy processor schematic is shown in Figure (7).

The $1^{\text {st }}$ input to the fuzzy processor is directly obtained from the INS mechanization and is measured by calculating the total body acceleration in G's. The second input is the jamming signal $\mathrm{J}_{\mathrm{S}}$ which is calculated using both values of carrier-to-noise power ratio $(\mathrm{C} / \mathrm{No})$ in clean and jammed environments $\left(\mathrm{C} / \mathrm{No}\right.$ and $[\mathrm{C} / \mathrm{No}]_{\mathrm{eq}}$ respectively). $\mathrm{C} / \mathrm{No}$ is calculated using the signal In-phase and Quadrature-phase (I\&Q) components as it explained in [7]. 


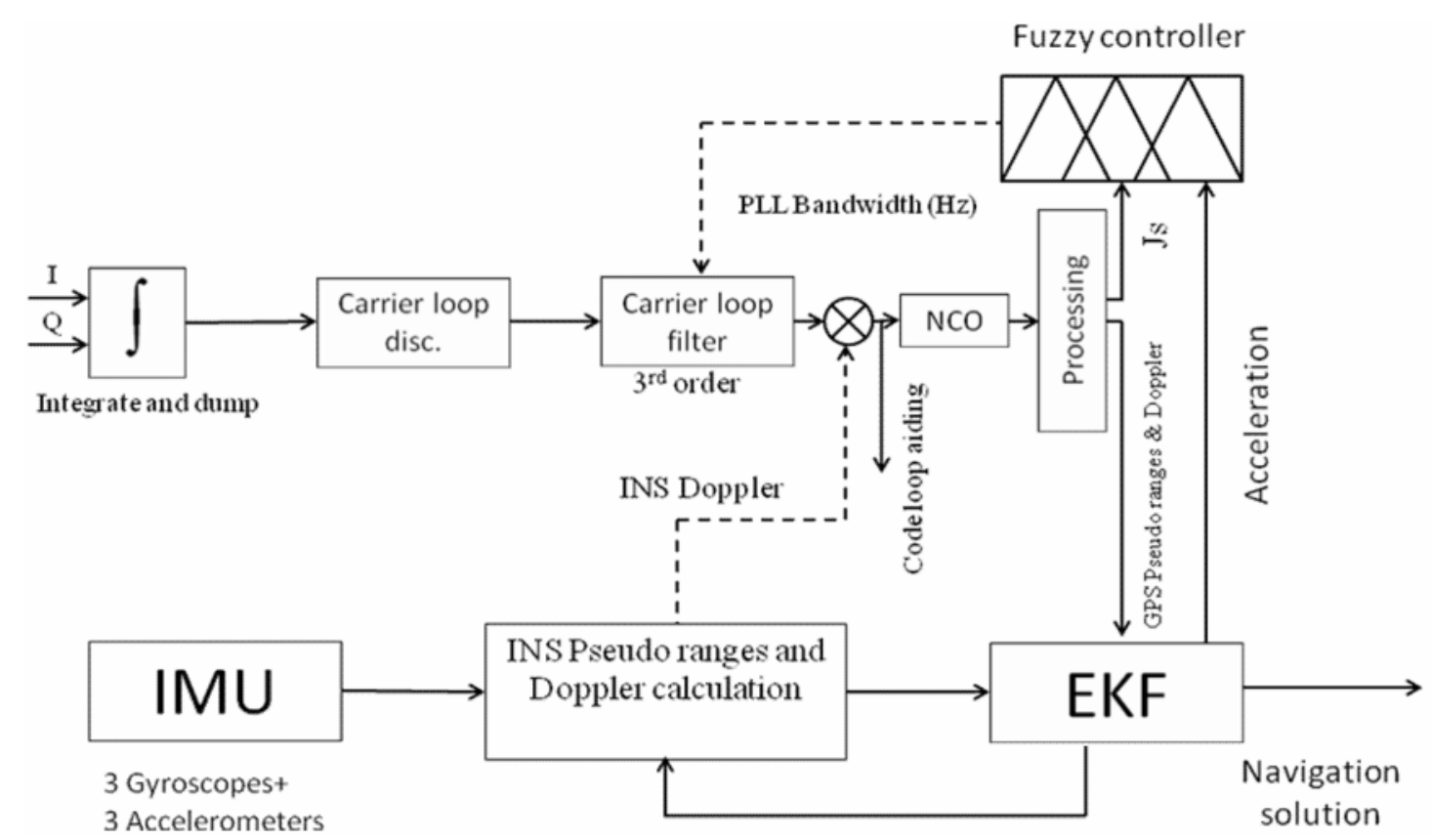

Figure (6): Adaptive Fuzzy Ultra Tight (AFUT) GPS/INS integration

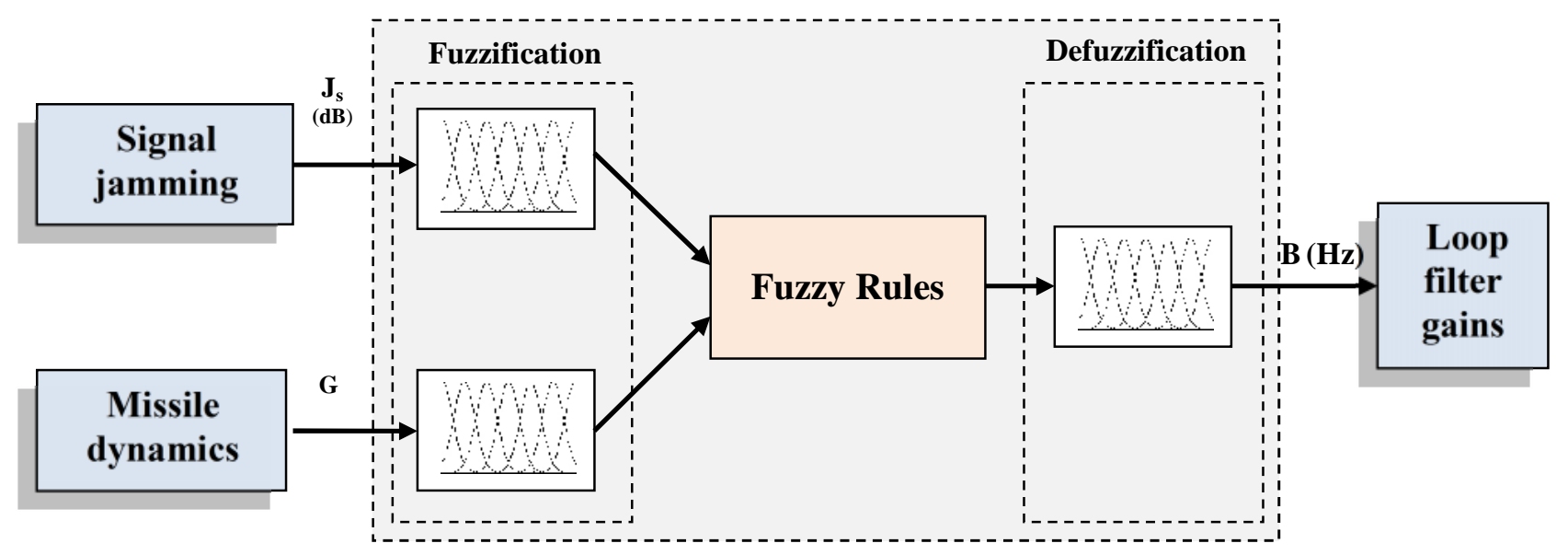

Figure (7): Bandwidth tuning fuzzy processor

Jamming-to-signal power ratio (JS) can be calculated as follows:

$$
J_{S}=10 \log \left[Q R_{c}\left(\frac{1}{10^{[C / N o]_{e q} / 10}}-\frac{1}{10^{[C / N o] / 10}}\right)\right](d B)
$$

where:

$\mathrm{C} / \mathrm{No}=$ unjammed carrier-to-noise power ratio in a $1 \mathrm{~Hz}$ bandwidth $(\mathrm{dB}-\mathrm{Hz})$ $[\mathrm{C} / \mathrm{No}]_{\mathrm{eq}}=$ jammed carrier-to-noise power ratio in a $1 \mathrm{~Hz}$ bandwidth $(\mathrm{dB}-\mathrm{Hz})$ 
Q $\quad=$ Spread spectrum processing gain adjustment factor

$\mathrm{R}_{\mathrm{c}} \quad=$ GPS PRN code chipping rate (chips/s)

Both jamming and acceleration inputs in addition to the required bandwidth output domains are designed and divided into several membership functions (MFs), each of them describes a certain linguistic definition of range (for example: Large, Medium, Low,...etc). The number of MFs adopted for the fuzzy controller is reported in Table (23).

Table (3): Distribution of fuzzy membership functions

\begin{tabular}{|l|l|c|}
\hline Type & Fuzzy Variable & Number of MFs \\
\hline Input(1) & Jamming (dB) & 5 \\
\hline Input(2) & Acceleration $(\mathrm{G})$ & 5 \\
\hline output & Bandwidth $(\mathrm{Hz})$ & 6 \\
\hline
\end{tabular}

The number of MFs associated with each fuzzy variable is a design parameter selected according to the required tracking accuracy, and the fuzzy rules that relate all the linguistic variables can be expressed as:

$\mathrm{R}_{\mathrm{i}}$ : If $\mathrm{x}_{1}$ is $\mathrm{A}_{1}^{i}$, and $\mathrm{x}_{2}$ is $\mathrm{A}_{2}^{i}$, then $\mathrm{y}$ is $\mathrm{B}^{\mathrm{i}} . \mathrm{i}=1,2 \ldots 54$

where $\mathrm{x}_{1}, \mathrm{x}_{2}$, and $\mathrm{y}$ are linguistic variables, and $\mathrm{A}_{1}{ }^{\mathrm{i}}, \mathrm{A}_{2}{ }^{\mathrm{i}}$ and $\mathrm{B}^{\mathrm{i}}$ are linguistic labels (or fuzzy sets) characterized by a MF. A defuzzification process is utilized to determine a crisp value according to the fuzzy output from the inference mechanism. The centreaverage method (Chen and Pham 2001), which calculates the centre of the area of the inference mechanism output possibility distribution, is used as defuzzification strategy. The output $\mathrm{y}$ is obtained as follows:

$\bar{y}=\frac{\sum_{i=1}^{n} y_{i} u\left(y_{i}\right)}{\sum_{i=1}^{n} u\left(y_{i}\right)}$

where $\mathrm{n}$ is the number of fuzzy output sets, $\mathrm{y}_{\mathrm{i}}$ is the numerical value of the $\mathrm{i}^{\text {th }}$ output membership function, and $\mathrm{u}\left(\mathrm{y}_{\mathrm{i}}\right)$ represents its membership value at the $\mathrm{i}^{\text {th }}$ quantization level. The fuzzy rule table providing the human knowledge base of the controller according to a deep investigation on dynamics and interference effects on tracking performance and relation with bandwidth is shown in Table (4).

The terms defined in Table (4) are: VL: Very low, L: Low, M: Medium, H: High, VH: Very high, ERR: Error. Gaussian MFs for inputs and triangular for output were chosen as shown in Figure (8), Figure (9), and Figure (10), respectively. MF parameters are 
chosen according to the designer experience and knowledge through a lot a performance tests under conditions of high dynamics and signal interference.

Table (4): Fuzzy rules

\begin{tabular}{|l|l|l|l|l|l|l|}
\hline $\mathrm{g} \downarrow$ & $\mathrm{JS} \rightarrow$ & VL & L & M & H & VH \\
\hline \hline VL & VL & VL & VL & VL & VL \\
\hline L & L & L & L & VL & VL \\
\hline M & M & M & L & L & Err \\
\hline H & H & M & M & Err & Err \\
\hline VH & VH & H & M & Err & Err \\
\hline
\end{tabular}

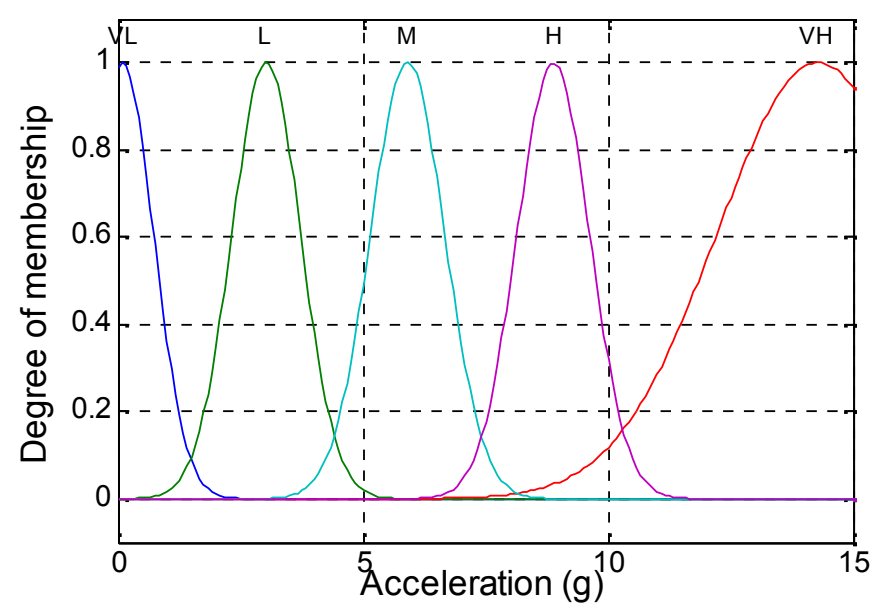

Figure (8): Acceleration membership function

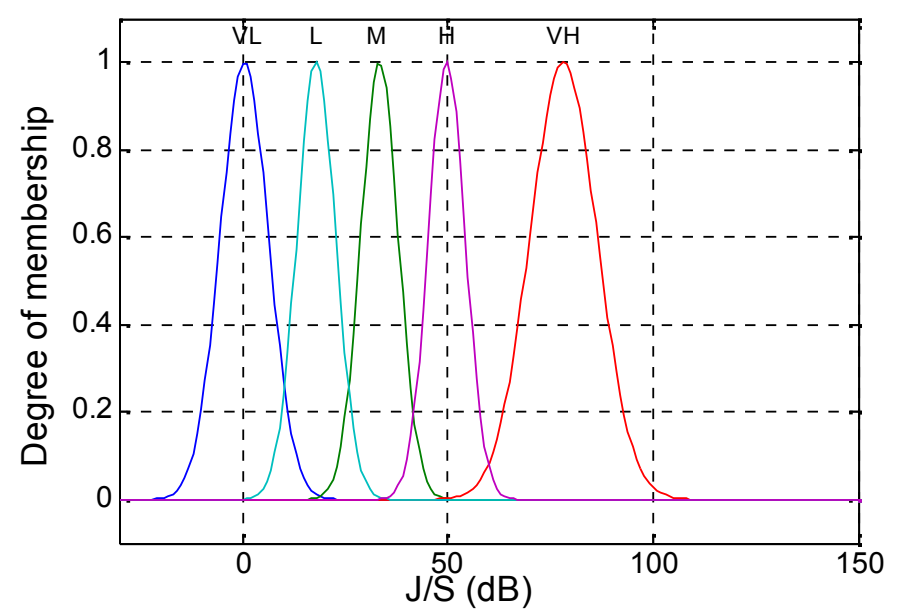

Figure (9): Jamming membership function 


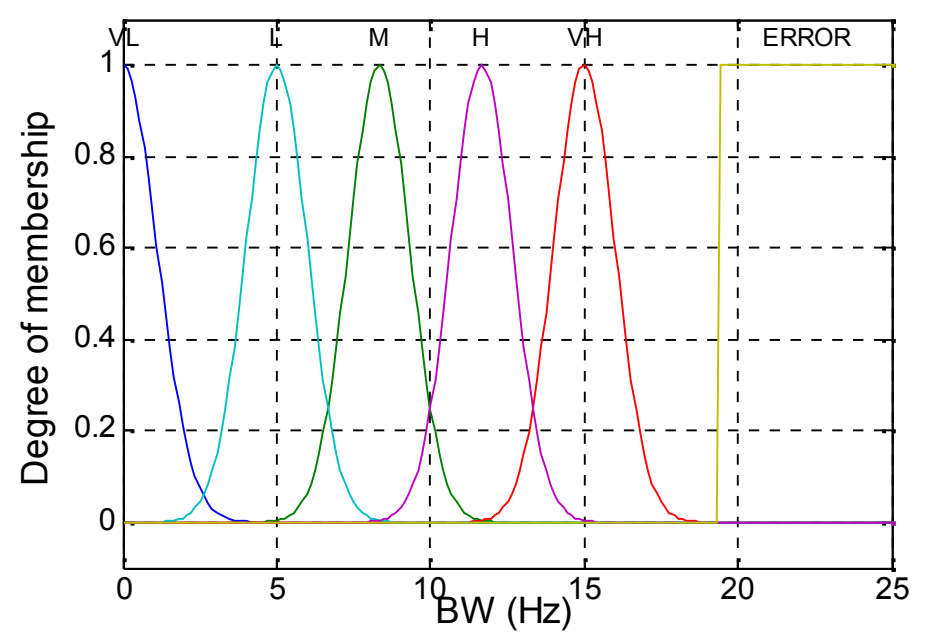

Figure (10): Bandwidth membership function

\section{Test Setup and Results}

The primary equipment used for testing the proposed algorithm, is the GPS GSS7700 hardware simulator from Spirent. Since high levels of dynamics are considered in this research, it is reasonable and easier if a hardware simulator is used for this purpose. The current hardware configuration is capable of producing GPS L1, L2, L5 frequencies, and IMU measurements, in addition to adjustable additive interference through two separate signal generators. IMU measurements errors are adjusted to match the low cost HG1700 IMU [9]. Several custom scenarios that present typical missile motion in space are designed and tested. The radio frequency (RF) signals are collected through an NI PXI 5661 front-end [10] after passing through an external low noise amplifier (LNA) using the specifications reported in Table (5, and saved for post processing. A software GPS receiver under MATLAB platform is used, in addition to $\mathrm{GSNRx}^{\mathrm{TM}}$ [11], the GNSS software receiver developed by the PLAN group at the University of Calgary which is used as a term of comparison. Although several scenarios have been considered, only the most illustrative results are reported here. Similar results have been obtained during the tests not reported in the paper.

Hardware configuration for the designed experiment is shown in Figure (11). The test scenario illustrated in Figure (12) shows the 2-D trajectory of a cruise missile. Its velocity, acceleration, and jerk profiles are shown in Figure (13). Jamming powers also increase at the periods marked 1:3 on Figure (12) as shown in 
Table (6). Points of high accelerations are designated by small letters (a, b ...e) on Figure (12) and Figure (13).

Table (5): Characteristics of the collected GPS signals

\begin{tabular}{|c|c|}
\hline Parameters & Value \\
\hline Sampling frequency & Fs $=5 \mathrm{MHz}$ \\
Intermediate frequency & FIF $=0.42 \mathrm{MHz}$ \\
Sampling & Complex \\
\hline
\end{tabular}

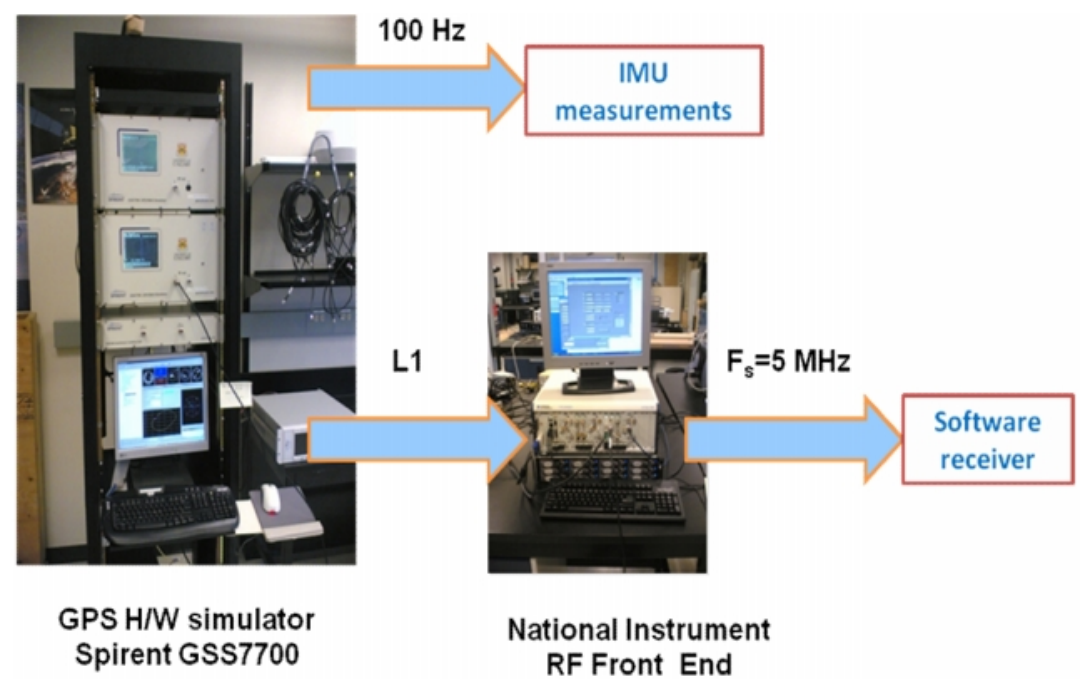

Figure (11): Hardware configuration

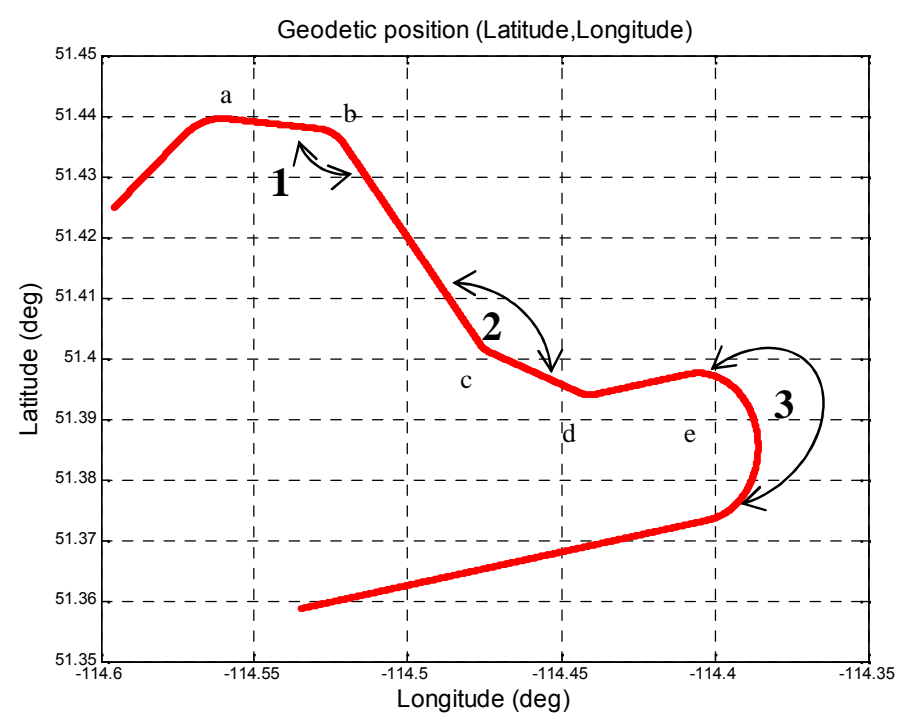

Figure (12): Test scenario includes different accelerations and jamming powers 
Table (6): Interference type and power through test trajectory

\begin{tabular}{|l|l|l|l|}
\hline Period & $\begin{array}{l}\text { Interference } \\
\text { type }\end{array}$ & $\begin{array}{l}\text { Power } \\
(\mathrm{dB})\end{array}$ & $\begin{array}{l}\text { Linguistic } \\
\text { definition }\end{array}$ \\
\hline 1 & Swept CW & 15 & Low \\
\cline { 4 - 5 } & \begin{tabular}{l} 
At 1575.42 \\
\cline { 3 - 4 }
\end{tabular} & 25 & moderate \\
\cline { 3 - 4 } & $\begin{array}{l}\mathrm{MHz} \pm \\
10 \mathrm{MHz}\end{array}$ & 35 & high \\
\hline
\end{tabular}
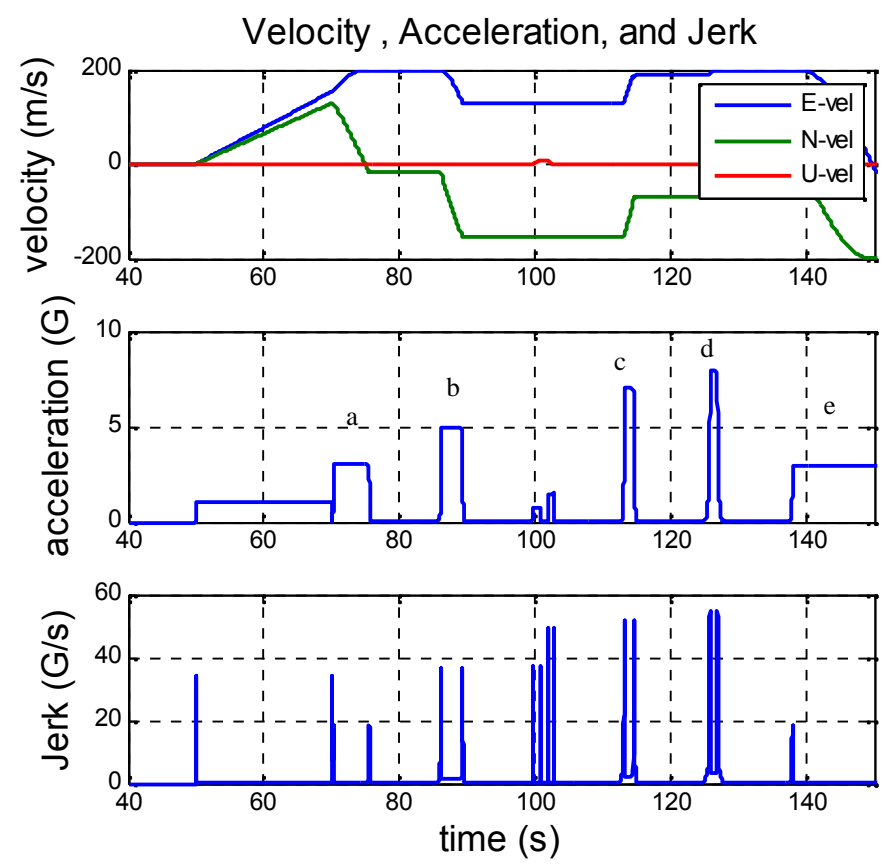

Figure (13): Velocities, accelerations, and jerks in the test scenario

The Fuzzy controller output is the desired tracking loop bandwidth. For this test the resultant bandwidth is shown in Figure (14). The bandwidth increases or decreases to weight between the receiver dynamics and signal interference. For extreme cases; for very high dynamics and interference situations, the controller decides to increase the bandwidth above the threshold value as an error indicator (Figure (10)) to the EKF to neglect the GPS measurements and rely only on the INS solution. The resultant system performance using the adaptive bandwidth filter gives very promising results much better than the stand alone GPS solution and even the integrated GPS/INS with a fixed gain. For the sake of comparison Figure (15) shows the GPS only solution position 
errors calculated by the GSNRx ${ }^{\mathrm{TM}}$ software receiver using a $4 \mathrm{~Hz}$ PLL with the interference and dynamics periods are indicated according to

Table (6), while Figure (16) shows the AFUT solution position errors.

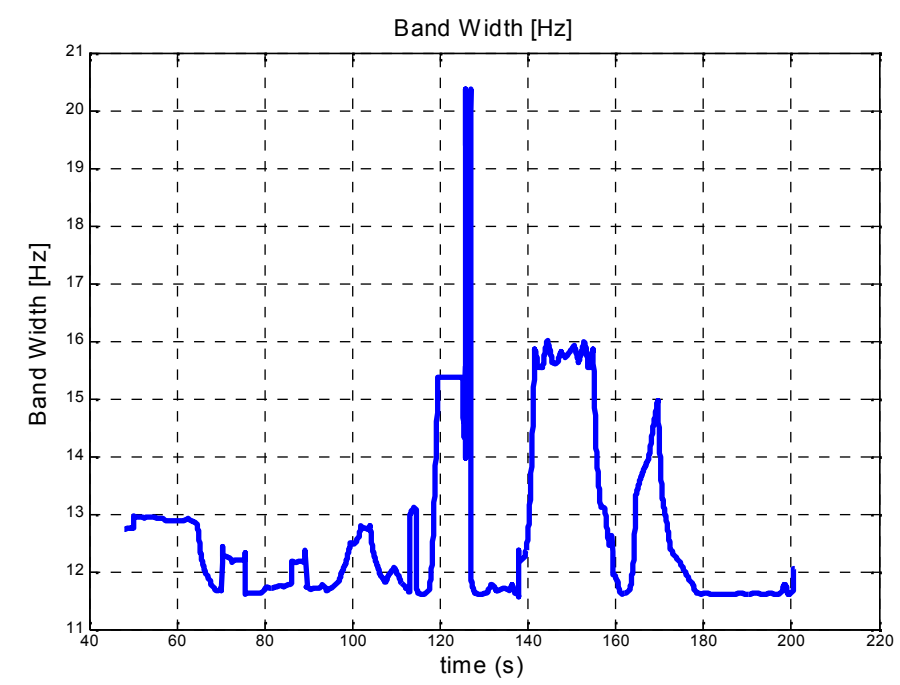

Figure (14): Calculated bandwidth for the test scenario

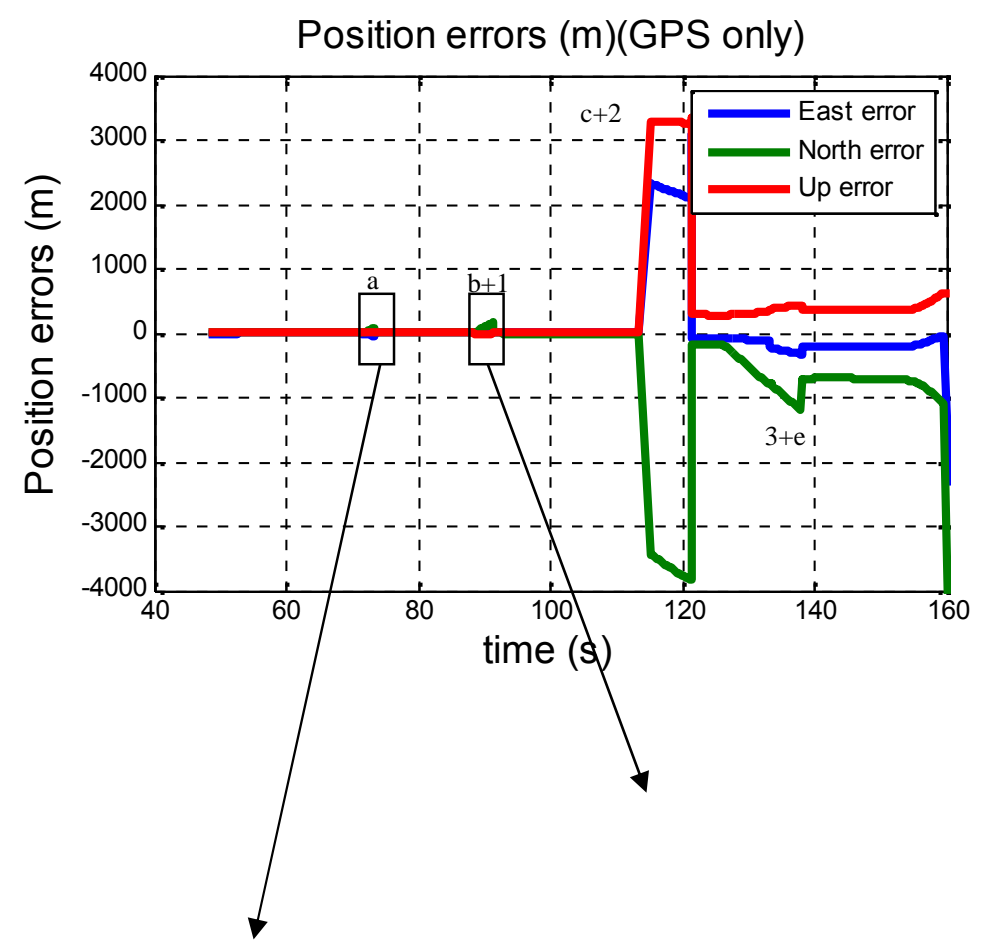




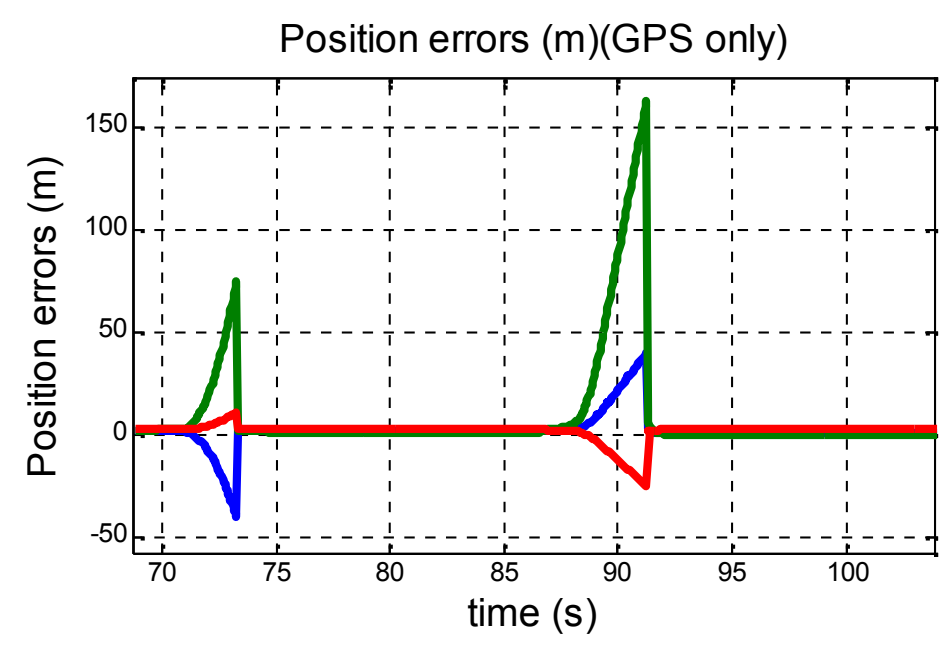

Figure (15): Effect of combined dynamics and interference on position errors using GPS only solution $(B W=4 \mathrm{~Hz})$

Position errors $(\mathrm{m})$

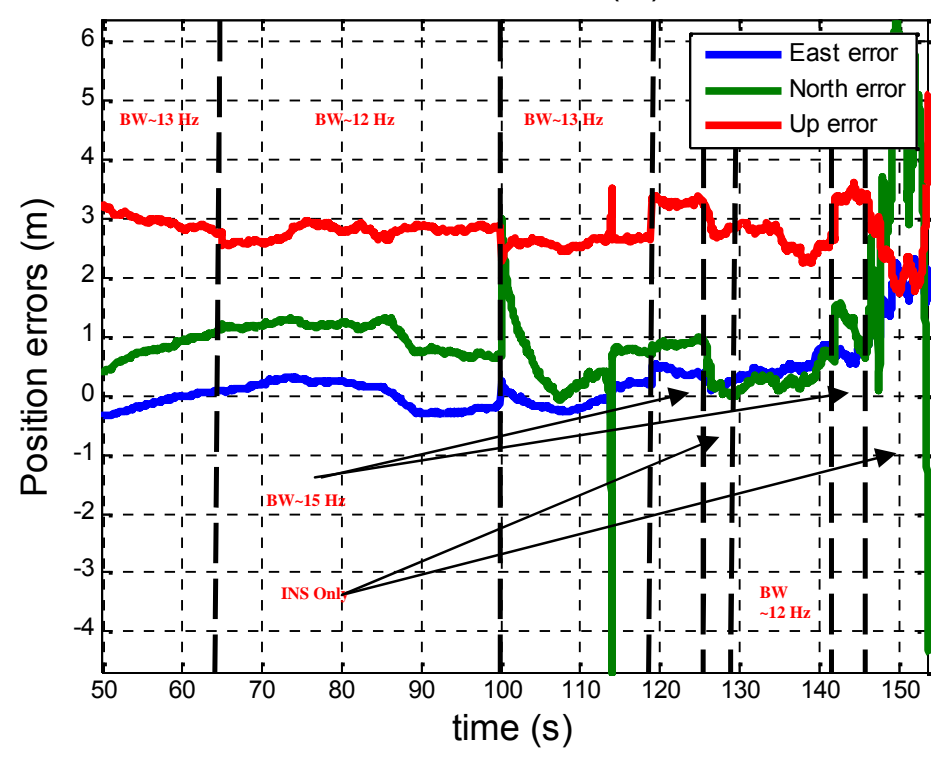

Figure (16): Effect of dynamics and interference on position errors using the proposed AFUT GPS/INS integration system solution

As it can be seen in Figure (16), the calculated position error using AFUT is much better than the one calculated using stand alone GPS. The horizontal position accuracy in northing and easting was around $1 \mathrm{~m}$, while vertical position accuracy was about $3 \mathrm{~m}$ which is slightly higher due to correlation between GPS pseudo ranges calculations and receiver clock error. By adapting the bandwidth, the PLL can combine between accuracy and dynamic performance. By aiding with INS through ultra tight integration, GPS receive guarantees continuous navigation solution and can be pulled back to the tracking mode by INS Doppler aiding if it loses lock due to high levels of interference or dynamics. 


\section{Conclusions:}

In this paper a new Adaptive Fuzzy Ultra Tight (AFUT) INS/GPS integration technique has been has been proposed and tested. The new system solves the contradiction between receiver bandwidth requirements using classical tracking techniques for either interference mitigation or dynamics tracking. The resultant system performance using AFUT gives very promising results much better than the stand alone GPS solution and even the integrated GPS/INS with a fixed gain.

\section{Acknowledgment:}

The author wishes to express his deep appreciation to his supervisor Prof. Gerard Lachapelle and his co-supervisor Prof. John Nelsen at the University of Calgary/ schulich school of engineering for their continuous support and guidance. Big thanks also to Dr. Danielle Borio from the PLAN group for giving valuable comments and directions.

\section{References:}

[1] A. Lawrence, Modern Inertial Technology: Navigation, Guidance, and Control. Austin, Texas, USA: Springer, 1998.

[2] D. H. Titterton and J. L. Weston, Strapdown Inertial Navigation Technology, 2nd Edition ed.: The Institution of Electrical Engineers \& The American Institute of Aeronautics and Astronautics, 2004.

[3] E. D. Kaplan, Understanding GPS: Principles and Applications, Second ed. Norwood, MA 02062: Artech House, INC., 2006.

[4] J. D. Gautier and B. W. Parkinson, "Using the GPS/INS Generalized evaluation Tool (GIGET) for the Comparison of Loosely Coupled, Tightly Coupled and Ultra-Tightly Coupled Integrated Navigation Systems," in Proceedings of ION 59th Annual Meeting/CIGTF 22nd Guidance Test Symposium Albuquerque, NM, 2003, pp. 65 - 76. 
[5] M. G. Petovello, C. O'Driscoll, and G. Lachapelle, "Ultra-Tight GPS/INS for Carrier Phase Positioning In Weak signal Environments," in NATO RTO SET-104 Symposium on Military Capabilities Enabled by Advances in Navigation Sensors Antalya, Turkey: NATO, 2007.

[6] J. Farrell, Aided Navigation: GPS with High Rate Sensors, First ed. vol. 1: Mcgraw-hill, 2008.

[7] M. S. Braasch and A. J. van Dierendonck, "GPS receiver architectures and measurements," in Proceedings of the IEEE. vol. 87: IEEE, 1999, pp. 4864.

[8] T. J. Ross, J. M. Booker, and W. J. Parkinson, Fuzzy Logic and Probability Applications. Alexandria, Virginia: American Statistical Association Society for Industrial and Applied Mathematics, 2002.

[9] Honeywell, "HG1700 Inertial Measurement Unit," 2006.

[10] National_Instruments, "2.7 GHz RF Vector Signal Analyzer with Digital Downconversion," Data Sheet: National Instruments (NI), 2006.

[11] C. O'Driscoll, D. Borio, M. Petovello, T. Williams, and G. Lachapelle, "The Soft Approach: A Recipe for a Multi-System, Multi-Frequency GNSS Receiver," InsideGNSS, vol. Volume 4, pp. pp. 46-51, 2009. 